\title{
Research and application based on MOOC courses certification platform
}

\author{
Bing Xu, Yizhi Zhang \\ Chongqing Three Gorges University, Chongqing, China
}

Keywords:MOOC, Certification platform, Framework,Asp.Net

\begin{abstract}
With the rapid development and application of Massive Open Online Courses (MOOC), new opportunities and challenges have been brought to the higher education. The MOOC platform has gradually released the course certificates to the course learners. In the paper, starting from the business and technical demand of the MOOC certification platform, the construction of the MOOC certification platform framework has been presented. By researches, the main function of MOOC certification system has been designed. The realization of the platform has extremely important application value and practical significance, which lays the foundation for the further researches and practices of MOOC.
\end{abstract}

\section{Introduction}

MOOC is a new type of course model, which has been occurred in the field of higher education since 2011. It is the free open online courses facing the public and is developed in the form of open educational resources. The year of 2012 was called the year of the MOOC by the New York Times [1]. It seems that MOOC has become the "digital tsunami" sweeping the global educational circle overnight and become the new thing that many universities have to research and deal with seriously [2]. MOOC indicates that in the field of higher education, a major reform is just around the corner. It challenges and overturns the traditional educational pattern and turns studying to be more active, convenient and personalized. It brings opportunities and challenges to the higher education in the aspect of spreading resources and signifies the most cutting-edge development trend of the higher education at present to some extent.

\section{Development of MOOC}

In the autumn of 2011, MOOC realized the first major breakthrough: over 160 thousand people participated in the artificial intelligence courses opened by Thorne and Peter Norwich in the Udacity Knowledge Laboratory newly founded by the professor Sebastian Thorne in Stanford University. In April, 2012, the two professors in the computer science of Stanford University released the website titled Coursera. 87 famous colleges and universities including Columbia University, Duke University and Princeton University have become the partners of the website successively. At present, more than 4.73 million people have registered and learned more than 440 courses in the website. In May, 2012, Massachusetts Institute of Technology (MIT) and Harvard University jointly launched edX. The program is based on the MITx program of MIT and the online teaching program of Harvard University, with the main aim to cooperate with the campus teaching, thus improving the teaching quality and promoting the online teaching. Within a short period of time, over 1 million learners have participated in the three MOOC platforms including Coursera, Udacity and edX to learn the free online courses. While continuously expanding three major American providers of MOOC, the European Union and Britain and so on, also follow the steps. In 
December, 2012, the British Open University cooperated with 12 colleges and universities in Britain to establish the MOOC platform named as "Future Learn". This program has been supported by British Council of Culture. Up to now, the program has 26 organization members and cooperative partners in the whole world. On April 25 ${ }^{\text {th }}, 2013$, the OpenupED of MOOC website jointly launched by 11 European countries was officially put online. The program wins the support of the European Commission [3]. At present, the domestic colleges and universities have launched the MOOC platform and course consortium in succession.

\section{Brief Introduction of MOOC Certification Platform}

At present, three major MOOC platforms have also released the course certificates to the course learners gradually. The colleges and universities taking part in the MOOC platform construction are more willing to recognize the MOOC and permit to transfer them into the credits of ordinary colleges and universities. The American public and private education certification bodies are also launching the examination and evaluation that whether MOOC has reached the level of colleges and universities. If the MOOC actually reaches the level of colleges and universities, they can be transferred to the credits, thus promoting the learners to take the course learning certification. While the design and application of MOOC certification platform shall be able to solve credit certification of MOOC [4]. This learning evaluation is not only conducive to preliminarily solving the credit certification problem in the course teaching of the colleges and universities in China, but also easy to solve the integrity of exams, which complies with the current situations of the higher education in China.

\section{Business and Technical Demand of MOOC Course Certification Platform}

According to the idea of the software engineering modeling, to construct any kind of platform framework patterns, there would be the business demand and the framework design. According to the idea of the MOOC pattern, the demand of platform business includes: firstly, Massive, which can support the unlimited number of learners; secondly, Open, which refers to that any person can $\log$ in and study in any place without the restrictions of the network environmental platform; thirdly, Online, which refers to the real-time implementation of the online course learning and communication as well as the in-time feedback of effects and evaluation; fourthly, Courses, which is the non-technical demand, requiring the online courses to be finely recorded, offer rich contents, have standard short videos and "fragmented" course resources and employ the lecturers with high teaching standard.

The technical demand of MOOC includes, firstly, Highly scalable, which supports a large number of registered students; secondly, Massive no. of concurrent users, which is great for sharing and supports the simultaneous online learning of a large number of users; thirdly, Good system response, which must guarantee that the users have the online learning experience; fourthly, Stability, which means that the network techniques must guarantee the logging in at any time; fifthly, Global access, which reflects openness and permits anybody to log in at any place; sixthly, Portability, which supports multiple terminals (especially the various cross-platform mobile terminals) and any browser and so on; seventhly, Security, which guarantees the integrity, confidentiality and availability of the systematic resources, especially the security of the students' course scores and the certification evaluation results, for which, it proposes higher requirements.

\section{Construction of MOOC Course Certification Platform Framework}


The construction of MOOC Certification Platform Technical Framework can apply the cloud computing platform overall framework, which is constituted by four layers including Data Layer for the storage of the various course resources and registered user information; Middle Layer for the data services, middleware and resource buffers and so on; Portal including the components of portal website, and interface tools on the front desk and so on; as well as Access Devices including various terminals[5], and mobile platforms and so on as shown in Figure 1.

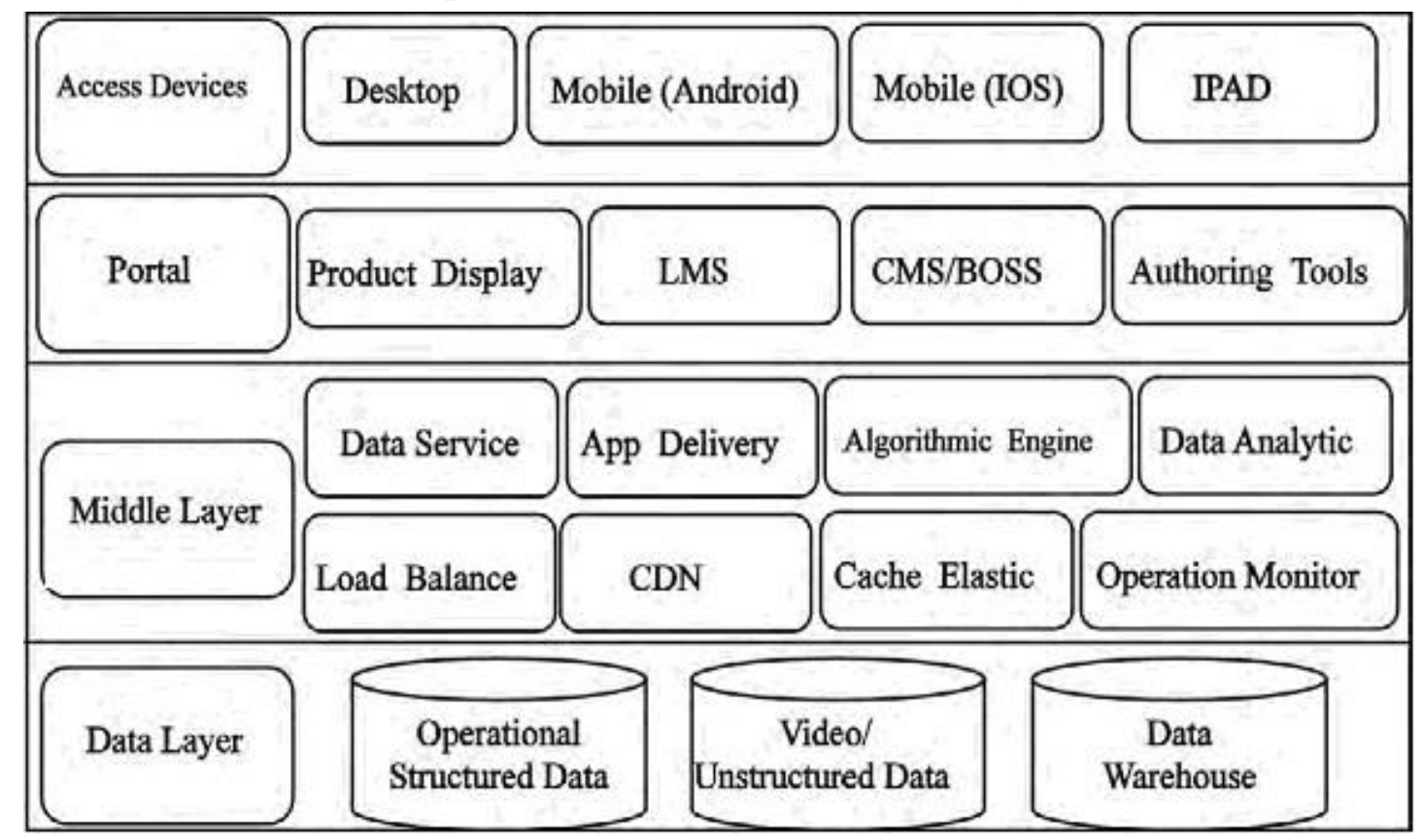

Figure 1: MOOC certification platform technical framework

\section{Main Functions of MOOC Course Certification Platform}

MOOC Course Certification Platform mainly adopts the technologies of Java, Asp.Net and Oracle and so on. The system mainly realizes the functions including question bank management, examinee management, marking teacher management, stochastic presentation of examination paper, examination management, marking management, query statistics, examination paper backup, scores management, and system management and so on As shown in Figure 2 The Structure Chart of Platform Functions) [6].

During usage, MOOC course certification platform generates the examination paper according to the parameters set by the administrator. The system will present the contents of the test questions automatically according to the arrangement requirements of the examination paper. The examinee shall participate in the exam by logging in the examination server after pressing in the student number and password. The backstage will read and appraise the examination paper automatically. After the completion of the examination and presenting the examination paper, the corresponding scores will be presented by the system. The administrator at the backstage can establish, browse, amend or delete the examination questions; or add, amend and delete the information of the marking teachers as well as the examinees; or conduct marking management, automatic marking, score statistics, score releasing, students' checking of the examination paper and the data backup and so on. 


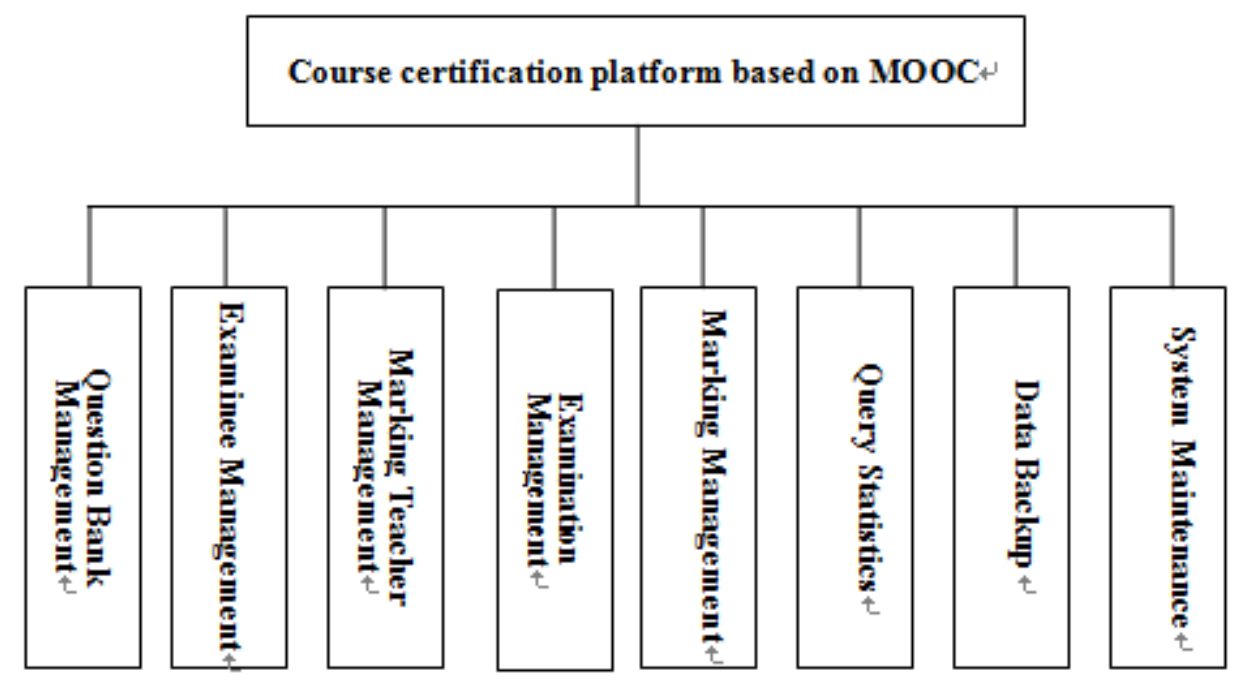

Figure 2: Structure chart of platform functions

\section{Design of MOOC Certification Platform}

Functional Module of the Platform

\section{Question Bank Management Subsystem}

It includes the functions in the aspects of question input, amendment and deletion. The question input includes the setting of the question properties and the designing of the question contents. They are mainly the single choices, true or false questions, multiple choices, gap filling and essay questions. It requires to have the contents with moderate difficulties, a wide coverage of knowledge points, strong representativeness and rational score allocation. And then, the questions are stored in the database of the backstage [7]. The question amendment can conduct query and amendment during the question input. When discovering mistakes during the question input, it shall amend the questions not complying with the standards in time. With the development of the society and the continuous deepening of the educational reform, the data in the question bank shall be renewed continuously and the backward and fault questions shall be deleted.

\section{Examinee Management Subsystem}

The examinee management subsystem can input the examinees needing the certification and amend the examination passwords of the examinees.

\section{Reading Teacher Management Subsystem}

The reading teacher management subsystem can input the information of the marking teachers in batches and amend the login passwords of the marking teachers.

\section{Exam Management Subsystem}

Exam management subsystem can set the examination time, time for the teachers to mark, question selection from the question bank as well as the optional examinations; and set the examinees participating in the examinations. In the meanwhile, it possesses the examination function and multiple practice function for students.

Marking Management Subsystem

The marking management subsystem can realize the automatic marking of objective questions, manual marking of subjective questions; set marking teachers and distribute examination paper. 


\section{Query Statistics Subsystem}

The query statistics subsystem realizes the score statistics of examinees, release and output of scores, students' checking of examination paper and students' query of scores.

Data Backup Subsystem

The data backup subsystem realizes the data backup for the examination paper of the students after the examination, marking and releasing of the scores, so that it could query for scores in the later period.

System Maintenance Subsystem

The system maintenance subsystem sets the basic information of the system and the configuration information of the database.

Design of the Database

During the research and development of MOOC certification platform, the design of database is of great importance. It must associate the functional requirements of the online examination system with the relevant theoretical knowledge of the database, so that to design the appropriate and efficient database. The design of the database must comply with the following requirements: the integrity, consistency and independence of the data, which must the guaranteed; having great reliability and security; being transplantable and scalable; and based on the design requirements of the database. It can divide the database into the following main relational schemas.

Users (user id, password, role of user, permission, name, gender and photo);

Administrator (administrator id, password and permission);

Question Bank (question id, type of question, content of question, answer of question, add time, difficulty level and question mark);

Course (course id, title of course, credit and type of course);

Student Answer (student no. id, question id, answer of examinee and note);

Examination Paper (examination paper id, course id, teacher id, question id, array, form of examination paper composition, quantity of questions, examination class and examination time);

Evaluation System (title of class, student no. id, name, scores of the first question type, scores of the second question type; scores of the third question type; scores of the fourth question type, scores of the fifth question type, total scores, marking teacher id).

\section{Conclusion}

The research and application based on the MOOC certification platform, not only provide conveniences for the credit certification during the MOOC studying, but also offer reference for the credit certification among colleges and universities, thus laying the foundation for the further research and practice of MOOC.

\section{Acknowledgment}

It is a project supported by the scientific fund assistance project from Chongqing Education Committee (NO: KJ1401027).

\section{References}

[1] Liu Jibin, Zhao Xiaoyu et al. Enlightenments Brought out by MOOC to the Teaching Reform of Chinese University Courses. Journal of Higher Education Research, 36, 4, 7-9(2013). 
[2] Ma Xinqiang, Huang Yi et al. Research on MOOC Education Platform Technology and Operation Mode. Chongqing Higher Education Research, 20,1,5-9(2014).

[3] Han Xibin, Ge Wenshuang et al. Comparative Research on MOOC Platform and Typical Network Teaching Platform. China Audio-Visual Education, 36,2,61-66(2014).

[4] Chi Yaqing, Song Ruiqiang et al. Exploration of the Influence of MOOC on Teaching of Computer Courses. Computer Engineering \& Science, 36,1,164-166(2014).

[5] Huang, J., Thinking on the integration of information technology and curricula. Modern Educational Technol., 15,3, 18-21 (2005).

[6] Zhao Wenmin, Hu Hua, Zhou Yi. Study on opening experimental teaching mode. Research and Exploration inLaboratory, 25, 9, 1116-1118 (2005).

[7] Zhou, M., Approaches to major optimization in higher vocational education from the perspective of industryadjustment with Zhejiang Province as an example. China Higher Educ. Research, 4, 97-100 (2012). 